\title{
Stable isotope analysis of diet-based social differentiation at late prehistoric collective burials in south-western portugal*
}

\author{
A. J. Waterman $\dagger$ \\ Department of anthropology, university of iowa, 114 macbride hall, iowa city, iowa 52242, usa
}

\section{R. H. Tykot}

Department of anthropology, university of south florida, tampa, fl 33620-7200, usa

And A. M. Silva

Department of life sciences, university of coimbra, coimbra, portugal 3001-401

In the neolithic and copper age collective burials of the portuguese estremadura, the majority of material culture and skeletal remains are highly commingled, making it difficult for archaeologists to evaluate social status by linking individuals with specific grave goods. In these circumstances, bio-anthropological data about individual life histories offer an addi- tional avenue of investigation into social complexity among prehistoric communities practis- ing collective burial. In this study, stable isotope data were gathered from 81 individuals from seven collective burial sites and one settlement, the fortified site of zambujal, in order to determine if significant dietary differences exist within or between burials that may point to patterns of social differentiation, both at individual sites and across the region. While in general all of the sampled individuals consumed fairly homogeneous diets based on terrestrial animal proteins and $c_{3}$ plants, this study found that statistically significant differences in $\delta^{13} c_{\text {ap }}$ and $\delta^{15} \mathrm{n}$ values exist between several sites, which may indicate socially differentiated consumption of meat and plants. Additionally, statistically significant differences were found in $\delta^{15} \mathrm{n}$ values between adults and juveniles, which may either be attributable to protein- restrictive child-feeding practices or physiological processes related to skeletal growth and development.

Keywords: portugal, stable isotope analysis, diet, social complexity, neolithic, copper age, collective burials

\section{NTRODUCTION}

In the Portuguese Estremadura during the Late Neolithic and Copper Age (LN/CA), there is archaeological evidence of the emergence of socially complex, chiefdom-scale, non-state societies (Lillios 1993; Gonçalves 1999; Cardoso 2007). Strategically located, fortified, hilltop settlements, such as Zambujal (c. 2800-1800 bc) (Sangmeister and Schubart 1981; Kunst 1995; Uerpmann and Uerpmann 2003), appear to have been centres of trade, craft production and 
population aggregation for these agrarian communities until their abandonment during the Bronze Age (Lillios 1993). It remains unclear how sociopolitical organization may have influenced the social collapse and settlement abandonment that occurred during the Early Bronze Age, as archaeologists have been unable to agree on the nature of social differentiation during the LN/CA, both in the Estremadura and in other regions of the Iberian Peninsula (Gonçalves 1994; Jorge 1995; Kunst 1995; Forenbaher 1999; Chapman 2003, 166-75; Chapman 2008; Díaz-delRío 2011). In general, there are three primary impediments to studies of social differ- entiation during this time. First, collective burial assemblages with commingled fragmentary skeletal remains and material objects are common in this region, making it difficult to distinguish social status from grave goods alone. Second, these collective burials often occur in different types of burial architecture (e.g., dolmens, natural and artificial caves, and tholoi), limiting our ability to relate tomb type and funerary-based labour investments to special social groups. Third, these burial spaces were used over hundreds of years, and comprise funerary assemblages that are a palimpsest of cultural activities, obscuring any temporally specific, individually directed burial rites that potentially could provide information about individual social statuses.

One promising avenue of investigation in this region is the use of bio-anthropological methodologies to study differences in biological markers between individuals at different burial sites (cf., Silva 2003; Cunha et al. 2007; Carvalho and Petchey 2013; Waterman et al. 2014a,b). Such investigations are beneficial because hard tissues store biological information pertaining to diet, activities, health and inter-lifetime mobility patterns - indicators that ethnographically and historically have been shown to correlate with social status and identity (cf., Owsley et al. 1987; Strickland and Shetty 1998; Farmer 2003). Bio-anthropological methods have been used effectively in archaeological research to identify status-related differences in diet, mobility and physical activity (cf., Schulting and Richards 2001; Le Huray and Schutkowski 2005; Bentley 2006; Price et al. 2006; Le Bras-Goude et al. 2013). However, some studies find little evidence of differences between skeletons from high-status and low-status burials (cf., Powell 1992; Cucina and I s s can 1997; Robb et al. 2001; Paine et al. 2007), illustrating that skeletal analysis cannot always open a window into past social relationships.

In this paper, we compare the results of stable isotope analysis of human and animal remains from seven LN/CA burial sites located near the settlement site of Zambujal, in the Portuguese Estremadura, to discern if any significant dietary differences exist between individuals interred at different burial sites that may point to patterns of social differentiation in the Zambujal region.

\section{STABLE ISOTOPE ANALYSIS}

Because it can provide a record of dietary patterns from prehistoric communities, stable isotope analysis has become a significant part of archaeological research. Many general reviews of stable isotope research have been published (cf., Schoeninger and Moore 1992; Ambrose and Krigbaum 2003; Katzenberg 2008) and the body of work related to this methodology continues to grow each year. The isotopes of carbon, nitrogen and oxygen are the most common stable isotopes analysed in archaeological research, but others, such as hydrogen and sulphur, are being used with increasing frequency (cf., Montgomery et al. 2005; Reynard and Hedges 2008).

Bone has an organic protein component, collagen, and a mineral component, apatite (hydroxyapatite). Generally, it is understood that the stable isotope ratios in bone collagen reflect dietary protein (Ambrose and Norr 1993; Richards and Hedges 1999; Tykot 2004), while ratios from bone apatite reflect the whole diet, including proteins, carbohydrates and 
lipids (Ambrose and Norr 1993; Schwarcz 2000; Tykot 2002, 2004). For collagen, calculations of the $\delta^{13} \mathrm{C}$ and $\delta^{15} \mathrm{~N}$ values are the two most common types of stable isotopic analysis completed in archaeological research, because they can quantify the dietary input of marine and terrestrial proteins, and the protein input of $\mathrm{C}_{3}$ and $\mathrm{C}_{4}$ (and CAM) plants (Smith and Epstein 1971; Chisholm et al. 1982; Ambrose and Norr 1993; Norr 1995; Tykot 2002, 216). In apatite, $\delta^{13} \mathrm{C}$ values are used to evaluate patterns of plant and animal consumption within the whole diet, and when $\delta^{13} \mathrm{C}$ data on both collagen and apatite are available, investigations of $\delta^{13} \mathrm{C}$ collagen-apatite spacing can provide a more nuanced understanding of dietary attributes. Since $\delta^{13} \mathrm{C}$ collagen $\left(\delta^{13} \mathrm{C}_{\mathrm{co}}\right)$ values represent dietary protein and the $\delta^{13} \mathrm{C}$ apatite $\left(\delta^{13} \mathrm{C}_{\mathrm{ap}}\right)$ values represent whole diet, differences in the spacing of these two values $\left(\delta^{13} \mathrm{C}_{\mathrm{co}}-\delta^{13} \mathrm{C}_{\mathrm{ap}}\right)$ are com- monly attributed to differential protein intake (Krueger and Sullivan 1984; Lee-Thorp et al. 1989). Comparisons of $\delta^{13} \mathrm{C}$ collagen and apatite can also be used to evaluate dietary intake of marine versus terrestrial protein and $C_{3}$ versus $C_{4}$ plants (Kellner and Schoeninger 2007). Oxygen isotope values $\left(\delta^{18} \mathrm{O}\right)$ provide information about water sources and can be used to distinguish between plants and animals from different geographical locations (Stuart-Williams et al. 1996; White et al. 2004).

In bone and tooth enamel, trophic-level adjustments in the values of $\delta^{15} \mathrm{~N}, \delta^{13} \mathrm{C}$ and $\delta^{18} \mathrm{O}$ also offer a way to investigate child-rearing and child-feeding practices, because breast milk provides an isotopically enriched food source and exclusively breastfed infants may exhibit as much as a $3 \%$ enrichment in nitrogen isotopic values over their mothers (Fogel et al. 1989; Wright and Schwarcz 1998). As breast milk consumption declines and supplemental foods are added, this enrichment slowly dissipates (Katzenberg et al. 1993; Fuller et al. 2006).

When investigating stable isotope ratios in skeletal remains, it is important to recognize that isotopic signatures reflect biological processes related to tissue formation. Bone continually remodels in response to growth, stress and strain, and calcium homeostasis. While different parts of the skeleton turn over at slightly different rates, it is generally accepted that isotopic values in bone reflect approximately the last 10 years of an individual's life (Manolagas 2000).

\section{SELECTED BURIAL SITES AND MATERIALS}

Human remains from 81 individuals were chosen from seven collective burial sites located within $25 \mathrm{~km}$ of the settlement site of Zambujal (Fig. 1). These represent different site types and their use-lives span an overlapping period of time from the LN/CA (3500-1800 bc) (Table 1). Because burials and settlements were geographically distinct at this time, they cannot be directly linked, but it is believed that burials located in the general proximity of settlements housed the remains of local peoples. The burials used in this study are as follows: (1) Cova da Moura (Spindler 1981; Silva 2003); (2) Feteira II (Horwath et al. 2014; Waterman et al.2014a);

(3) Lapa da Rainha II (Kunst and Trindade 1990); (4) Bolores (Kunst and Trindade 1990; Lillios et al. 2010); (5) Cabeço da Arruda I (Trindade and da Veiga Ferreira 1956; Silva 2002, 2003); (6) Paimogo I (Tholos de Pai Mogo I) (Gallay et al. 1973; Silva 2002, 2003); and (7) Tholos da Borracheira (Leisner 1965; Kunst and Trindade 1990; and see Waterman 2012 for additional information). A small amount of skeletal remains recovered from Zambujal was also analysed. Although less common, occasional caches of human remains are recovered from settlements. The reasons for these settlement burials are unclear, but preliminary analyses of the temporal and spatial contexts of these finds are under way (Kunst et al. 2012). Faunal samples from five of the sites were also analysed to ascertain the variability of baseline isotopic signa- tures in the region. 


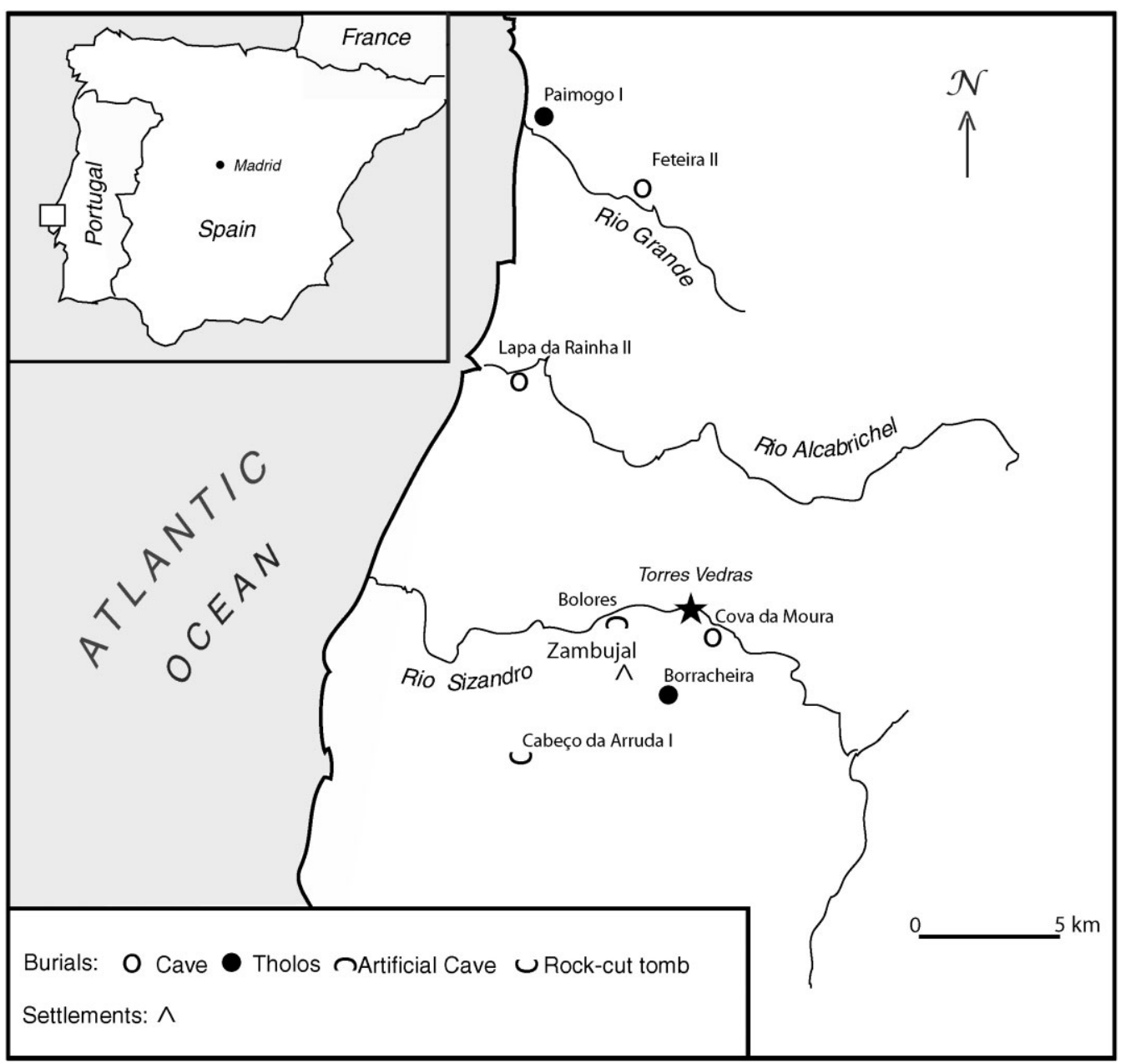

Figure 1 A map of selected sites (adapted from Waterman et al. 2014b).

\section{METHODS}

As the human remains were recovered from commingled burials, overlapping cranial and/ mandibular fragments were used to distinguish individuals. Animal samples were obtained from both cranial and post-cranial elements. Samples were sent to the Laboratory for Archaeological Science at the University of South Florida (USF) for further processing, where collagen and apatite samples were obtained using the techniques described in Tykot (2006). All collagen yields and $\mathrm{C}: \mathrm{N}$ ratios in collagen were measured, as yields of less than $1 \%$ have been found to be too degraded for reliable analysis, and $\mathrm{C}: \mathrm{N}$ ratios between 3.7 and 2.9 are generally found to indicate good preservation (DeNiro 1985; Tykot 2002). In order to remove any non-biogenic carbonates that may have leached into the bone apatite from the burial environment, all bone apatite was pre-treated with a buffered acetic acid solution. Such treatments have been found effective in removing post mortem contamination while retaining the in vivo isotopic signature (Koch et al. 1997; Tykot 2002). The samples were analysed for carbon, nitrogen and oxygen 
Table 1 Burial site type and chronology

\begin{tabular}{|c|c|c|c|c|c|}
\hline Site & Burial site type & Laboratory number & Date (BP) & Cal $B C 2 \sigma$ & Reference \\
\hline Feteira II & Natural cave & Sac-1563 & $4760 \pm 80$ & $3694-3367$ & Waterman (2006) \\
\hline Feteira II & Natural cave & Sac-1562 & $4370 \pm 45$ & $3264-2894$ & Waterman (2006) \\
\hline Cova da Moura & Natural cave & UBAR-593 & $4715 \pm 50$ & $3635-3372$ & Silva (2002) \\
\hline Cova da Moura & Natural cave & UBAR-536 & $3950 \pm 60$ & $2620-2212$ & Silva (2002) \\
\hline Tholos da Borracheira & Tholos & UGAMS 8455 & $4420 \pm 25$ & $3310-2923$ & Waterman (2012) \\
\hline Tholos da Borracheira & Tholos & UGAMS 8454 & $3720 \pm 25$ & 2199-2035 & Waterman (2012) \\
\hline Cabeço da Arruda I & Rock-cut tomb & Beta-123363 & $4370 \pm 70$ & $3331-2885$ & Silva (2002) \\
\hline Cabeço da Arruda I & Rock-cut tomb & Beta-132975 & $4240 \pm 50$ & $3005-2634$ & Silva (2002) \\
\hline Paimogo I & Tholos & Sac-1556 & $4259 \pm 90$ & $3264-2576$ & Silva (2002) \\
\hline Paimogo I & Tholos & UBAR-539 & $4139 \pm 90$ & $2901-2488$ & Silva (2002) \\
\hline Bolores & Artificial cave & Beta-249032 & $4150 \pm 40$ & $2880-2620$ & Lillios et al. (2010) \\
\hline Bolores & Artificial cave & Beta-235487 & $3530 \pm 40$ & $1963-1745$ & Lillios et al. (2010) \\
\hline Lapa da Rainha II & Natural cave & UGAMS 8453 & $4080 \pm 25$ & $2852-2497$ & Waterman (2012) \\
\hline Zambujal & Settlement & KIA-27565 & $4445 \pm 31$ & $3333-2936$ & Kunst and Lutz (2008) \\
\hline Zambujal & Settlement & GrN-6668 & $3625 \pm 65$ & $2198-1776$ & Kunst and Lutz (2008) \\
\hline
\end{tabular}


isotopes using a CHN analyser coupled with a Finnigan MAT Delta Plus stable isotope ratio mass spectrometer, using continuous flow, for the collagen; and a Finnigan MAT Delta Plus instrument, using a Kiel III device with $100 \%$ phosphoric acid $\left(\mathrm{H}_{3} \mathrm{PO}_{4}\right)$ at $90^{\circ} \mathrm{C}$, for the apatite.

The carbon, nitrogen and oxygen ratios are reported using the delta $(\delta)$ notation and calibrated to an international standard using the following standard formula: $\delta=\left[\left(R_{\text {sample }} / R_{\text {standard }}\right)-1\right) \times$ $1000]$, where $R_{\text {sample }}$ is the ratio of the heavy isotope to light isotope in the ake.g., ${ }^{13} \mathrm{C} /{ }^{12} \mathrm{C}$ or $\left.{ }^{15} \mathrm{~N} /{ }^{14} \mathrm{~N}\right), R_{\text {standard }}$ is the ratio of the heavy isotope to light isotope in the standard and $\delta$ is the difference in isotopic composition of the sample relative to that of the reference, expressed in parts per thousand (\%o) (see Tykot 2006). In this study, the $\delta^{13} \mathrm{C}, \delta^{15} \mathrm{~N}$ and $\delta^{18} \mathrm{O}$ notations are calculated using Ambient Inhalable Reservoir (AIR) and the Pee Dee Belemnite (PDB) standards. The analytical precision of the employed instrument was $\pm 0.1 \%$ for the $\delta^{13} \mathrm{C}$ data and $\pm 0.2 \%$ for the $\delta^{15} \mathrm{~N}$ and $\delta^{18} \mathrm{O}$ data.

To test if statistically significant $(P<0.05)$ differences in the dietary data existed between burial sites, one-way analysis of variance (ANOVA) and the Kruskal-Wallis test were employed to compare the interval data sets for Cova da Moura, Feteira II, Bolores, Cabeço da Arruda I and Paimogo I. The other three sites were excluded from the statistical tests because of insufficient sample sizes. All statistical calculations were completed using NCSS 2003 software. RESULTS

\section{Stable isotope analysis}

Collagen data were successfully obtained for 20/22 sampled animals and 74/81 humans. Bone collagen was too degraded in the remaining seven humans and two animals to be properly analysed. Bone apatite results were acquired for 21/22 sampled animals and 77/81 humans. For one pig and four humans, no data were obtained due to machine error (Tables 2 and 3; Figs 2 and 3).

\section{Statistical tests}

When the data were compared using either one-way ANOVA or the Kruskal-Wallis test, statistically significant differences between sites were found for two of the four tested variables (see supplementary Tables S1-S5). One-way ANOVA was used for the $\delta^{13} \mathrm{C}_{\mathrm{co}}$ and $\delta^{15} \mathrm{~N}$ data because assumption tests proved normal distribution and equal variance. While no statistically significant differences were found for the $\delta^{13} \mathrm{C}_{\mathrm{co}}$ data, statistically significant differences in $\delta^{15} \mathrm{~N}$ values were exhibited by at least one site $(p=0.0026)$. When the post-hoc Tukey-Kramer MultipleComparison test was run, statistically significant differences in $\delta^{15} \mathrm{~N}$ values were found between Cova da Moura and Feteira II ( $p=0.000169)$ and Cova da Moura and Paimogo I $(p=0.019746)$.

The non-parametric Kruskal-Wallis test was used for the $\delta^{13} \mathrm{C}_{\text {ap }}$ and $\delta^{18} \mathrm{O}$ data as these data violated the assumptions of normal distribution and equal variance. Statistically significant differences were detected for the $\delta^{13} \mathrm{C}_{\mathrm{ap}}$ data between sites $(p=0.000028)$, but not for the $\delta^{18} \mathrm{O}$ data. Mann-Whitney $U$ tests showed that the statistically significant differences were found between Cova da Moura and four of the other sites, these being Paimogo I $(p=0.00033)$, Feteira II $(p=0.00085)$, Cabeço da Arruda I ( $p=0.00014)$ and Bolores $(p=0.00029)$. Additionally, statistically significant differences in the $\delta^{13} \mathrm{C}_{\mathrm{ap}}$ data were found between Bolores and Feteira II $(p=0.02626)$.

Statistical tests were also used to compare the stable isotope data by age-grade for all sites. While no differences were found for the $\delta^{13} \mathrm{C}_{\mathrm{co}}, \delta^{13} \mathrm{C}_{\mathrm{ap}}$ and $\delta^{18} \mathrm{O}$ using the Kruskal-Wallis test, 
Table 2 Results, fauna

\begin{tabular}{|c|c|c|c|c|c|c|c|c|c|}
\hline Site & Sample & Genus & USF laboratory number & $\delta^{13} C_{c o}$ & $\delta^{15} N$ & $C: N$ & USF laboratory number & $\delta^{13} C_{a p}$ & $\delta^{18} \mathrm{O}$ \\
\hline Zambujal & z829 & Bos & 13710 & -20.5 & 5 & 3.3 & 13733 & -12.5 & -1.7 \\
\hline Zambujal & z812 & Bos & 13711 & & & & 13734 & -12.0 & -1.3 \\
\hline Zambujal & z1128 & Bos & 13712 & -20.4 & 8.1 & 3.4 & 13735 & -12.9 & -0.5 \\
\hline \multirow[t]{3}{*}{ Zambujal } & z10000 & Bos & 13713 & -20.3 & 6.1 & 3.4 & 13736 & -12.7 & -1.6 \\
\hline & & & Average & -20.4 & 6.4 & & Average & -12.5 & -1.3 \\
\hline & & & sd & 0.1 & 1.6 & & $\mathrm{sd}$ & 0.4 & 0.5 \\
\hline Zambujal & z142 & Sus & 13714 & -21.4 & 10.5 & 3.2 & 13737 & -15.6 & -8.6 \\
\hline Zambujal & z197 & Sus & 13715 & -19.8 & 4.4 & 3.4 & 13738 & -11.2 & -2.0 \\
\hline Zambujal & z159 & Sus & 13716 & -20.5 & 6.3 & 3.3 & 13739 & -14.1 & -2.4 \\
\hline Zambujal & z344 & Sus & 13717 & -20.6 & 7.3 & 3.2 & 13740 & -12.8 & -2.9 \\
\hline Zambujal & z87103 & Sus & 13718 & -19.8 & 5.2 & 3.2 & 13741 & -12.3 & -1.6 \\
\hline Zambujal & z8743409 & Sus & 13725 & -21.1 & 6.5 & 3.4 & 13748 & & \\
\hline Zambujal & z87103403 & Sus & 13726 & -20.9 & 5.7 & 3.4 & 13749 & -11.7 & -4.4 \\
\hline \multirow[t]{3}{*}{ Cova da Moura } & $\mathrm{CM} \mathrm{f3}$ & Sus & 13727 & -20.1 & 10.6 & 3.3 & 13750 & -13.3 & -1.5 \\
\hline & & & Average & -20.5 & 7.1 & & Average & -13.0 & -3.3 \\
\hline & & & sd & 0.6 & 2.3 & & sd & 1.5 & 2.5 \\
\hline Zambujal & z951 & Cervus & 13719 & -20.7 & 4.7 & 3.2 & 13742 & -11.5 & -2.6 \\
\hline \multirow[t]{3}{*}{ Zambujal } & z1041 & Cervus & 13720 & -20.9 & 4.4 & 3.4 & 13743 & -10.7 & -2.1 \\
\hline & & & Average & -20.8 & 4.6 & & Average & -11.1 & -2.4 \\
\hline & & & $\mathrm{sd}$ & 0.1 & 0.2 & & sd & 0.6 & 0.4 \\
\hline Zambujal & z469 & Ovis/Capra & 13721 & -21.2 & 6.0 & 3.4 & 13744 & -11.9 & -1.9 \\
\hline Zambujal & z492 & Ovis/Capra & 13722 & -20.5 & 5.7 & 3.2 & 13745 & -11.4 & -1.7 \\
\hline Zambujal & z1136 & Ovis/Capra & 13723 & -20.6 & 5.1 & 3.5 & 13746 & -9.7 & -2.7 \\
\hline \multirow[t]{3}{*}{ Zambujal } & z87101 & Ovis/Capra & 13724 & -20.5 & 5.4 & 3.2 & 13747 & -13.3 & -1.7 \\
\hline & & & Average & -20.7 & 5.6 & & Average & -11.6 & -2 \\
\hline & & & sd & 0.3 & 0.4 & & sd & 1.5 & 0.5 \\
\hline Paimogo I & PM f1 & Oryctolagus & 12762 & & & & 12778 & -14 & -1.6 \\
\hline Paimogo I & PM f2 & Oryctolagus & 12763 & -22.1 & 3.8 & 3.2 & 12779 & -13.2 & -0.9 \\
\hline Cova da Moura & CM f1 & Oryctolagus & 12838 & -22.9 & 3.6 & 3.3 & 12870 & -11.0 & -0.5 \\
\hline \multirow[t]{3}{*}{ Lapa da Rainha II } & LR f1 & Oryctolagus & 12839 & -21.8 & 4.7 & 3.3 & 12871 & -10.7 & -2.1 \\
\hline & & & Average & -22.3 & 4.0 & & & -12.2 & -1.3 \\
\hline & & & $\mathrm{sd}$ & 0.6 & 0.6 & & & 1.6 & 0.7 \\
\hline
\end{tabular}


Table 3 Result, humans

\begin{tabular}{|c|c|c|c|c|c|c|c|c|c|c|c|}
\hline Site & $\begin{array}{l}\text { Catalogue } \\
\text { number }\end{array}$ & Individual & $A g e^{*}$ & $\begin{array}{c}\text { USF laboratory } \\
\text { number }\end{array}$ & $\delta^{13} C_{c o}$ & $\delta^{15} N$ & $C: N$ & $\begin{array}{c}\text { USF laboratory } \\
\text { number }\end{array}$ & $\delta^{13} C_{a p}$ & $\delta^{18} O_{a p}$ & $\delta^{13} C_{c o-a p}$ \\
\hline Cabeca da Arruda I & CAI 13 & Adult 1 & Adult & 13818 & -19.5 & 8.5 & 3.2 & 13835 & -13.4 & -1.9 & 6.1 \\
\hline Cabeca da Arruda I & CAI 11 & Adult 2 & Adult & 13820 & -19.8 & 8.6 & 3.3 & 13837 & -13.7 & -2.8 & 6.1 \\
\hline Cabeca da Arruda I & CAI 17 & Adult 3 & Adult & 13819 & -19.5 & 8.4 & 3.3 & 13836 & -13.5 & -2.6 & 6.0 \\
\hline Cabeca da Arruda I & CAI 1 & Adult 4 & Adult & 13816 & -19.6 & 8.8 & 3.3 & 13833 & -13.2 & -2.3 & 6.4 \\
\hline Cabeca da Arruda I & CAI 25 & Adult 5 & Adult & 13817 & -19.8 & 9.7 & 3.3 & 13834 & -14.1 & -3.3 & 5.7 \\
\hline Cabeca da Arruda I & CAI 3 & Adult 6 & Adult & 13822 & -20.3 & 8.6 & 3.2 & 13839 & -13.4 & -2.0 & 6.9 \\
\hline Cabeca da Arruda I & CAI 7 & Adult 7 & Adult & 13821 & -19.8 & 7.8 & 3.3 & 13838 & & & \\
\hline Cabeca da Arruda I & CAI 2 & Juvenile 1 & $8-10 \mathrm{yr}$ & 13823 & -20.3 & 7.8 & 3.4 & 13840 & -14.0 & -2.6 & 6.1 \\
\hline Cabeca da Arruda I & CAI 23 & Juvenile 2 & $8-10 \mathrm{yr}$ & 13824 & -20.0 & 9.0 & 3.3 & 13841 & -13.9 & -2.7 & 6.3 \\
\hline Cabeca da Arruda I & CAI 19 & Juvenile 3 & $10-15 \mathrm{yr}$ & 13815 & -20.1 & 8.5 & 3.3 & 13832 & -14.3 & -1.7 & 5.8 \\
\hline Zambujal & z831 & Adult 1 & Adult & 13730 & -20.7 & 8.4 & 3.2 & 13753 & -12.4 & -2.0 & 8.3 \\
\hline Zambujal & z776 & Adult 2 & Adult & 13731 & -20.1 & 10.1 & 3.3 & 13754 & -12.6 & -1.8 & 7.5 \\
\hline Zambujal & z971 & Adult 3 & Adult & 13732 & & & & 13755 & & & \\
\hline Zambujal & $\mathrm{z} 521$ & Juvenile 1 & $0-1 \mathrm{yr}$ & 13729 & -18.9 & 11.7 & 3.3 & 13752 & & & \\
\hline Zambujal & z895 & Juvenile 2 & $5-10 \mathrm{yr}$ & 13728 & -20.0 & 8.6 & 3.4 & 13751 & -6.1 & -1.4 & 13.9 \\
\hline Paimogo I & PM12268 & Adult 1 & Adult & 12749 & -21.3 & 8.1 & 3.2 & 12765 & -13.4 & -3.0 & 7.9 \\
\hline Paimogo I & PM12263 & Adult 2 & Adult & 12750 & -21.0 & 10.3 & 3.2 & 12766 & -12.9 & -2.7 & 8.1 \\
\hline Paimogo I & PM12261 & Adult 3 & Adult & 12751 & -20.4 & 9.3 & 3.2 & 12767 & -13.6 & -2.8 & 6.8 \\
\hline Paimogo I & PM12338 & Adult 4 & Adult & 12752 & -20.8 & 8.5 & 3.3 & 12768 & -14.5 & -3.3 & 6.3 \\
\hline Paimogo I & PM12951 & Adult 5 & Adult & 12754 & -19.8 & 8.8 & 3.2 & 12770 & -12.2 & -2.1 & 7.6 \\
\hline Paimogo I & PM12282 & Adult 6 & Adult & 12755 & -20.1 & 8.5 & 3.2 & 12771 & & & \\
\hline Paimogo I & PM12821 & Adult 7 & Adult & 12756 & -20.0 & 8.0 & 3.3 & 12772 & -14.7 & -3.0 & 5.3 \\
\hline Paimogo I & PM12600 & Adult 8 & Adult & 12757 & -20.3 & 8.1 & 3.2 & 12773 & -13.4 & -2.9 & 6.9 \\
\hline Paimogo I & PM12867 & Juvenile 1 & $5-7 \mathrm{yr}$ & 12758 & -20.5 & 7.4 & 3.2 & 12774 & -13.4 & -2.5 & 7.1 \\
\hline Paimogo I & PM12906 & Juvenile 2 & $3-5 \mathrm{yr}$ & 12759 & -20.1 & 8.2 & 3.2 & 12775 & -12.6 & -2.4 & 7.5 \\
\hline Paimogo I & PM12507 & Juvenile 3 & $3-5 \mathrm{yr}$ & 12761 & -19.7 & 8.6 & 3.2 & 12777 & -12.4 & -3.6 & 7.3 \\
\hline Paimogo I & PM12783 & Juvenile 4 & $15-25 \mathrm{yr}$ & 12760 & -19.7 & 7.8 & 3.2 & 12776 & -13.9 & -1.7 & 5.8 \\
\hline Paimogo I & PM12726 & Juvenile 5 & $12-16 \mathrm{yr}$ & 12753 & -20.0 & 7.9 & 3.2 & 12769 & -14.4 & -3.5 & 5.6 \\
\hline
\end{tabular}




\begin{tabular}{|c|c|c|c|c|c|c|c|c|c|c|c|}
\hline Paimogo I & PM12267 & Juvenile 6 & $15-25 \mathrm{yr}$ & 12748 & -19.7 & 10.1 & 3.2 & 12764 & -12.6 & -2.1 & 7.1 \\
\hline Feteira II & Fet 1222 & Adult 1 & Adult & 12780 & -20.2 & 8.4 & 3.2 & 12794 & -10.9 & -1.1 & 9.3 \\
\hline Feteira II & Fet 1219 & Adult 2 & Adult & 12781 & -19.9 & 8.6 & 3.2 & 12795 & -14.0 & -3.7 & 5.9 \\
\hline Feteira II & Fet 1547 & Adult 3 & Adult & 12782 & -20.6 & 7.5 & 3.2 & 12796 & -13.8 & -0.8 & 6.8 \\
\hline Feteira II & Fet 342 & Adult 4 & Adult & 12783 & -20.4 & 8.6 & 3.2 & 12797 & -12.2 & -2.0 & 8.2 \\
\hline Feteira II & Fet 944 & Adult 5 & Adult & 12784 & -20.1 & 8.2 & 3.2 & 12798 & -13.5 & -3.3 & 6.6 \\
\hline Feteira II & Fet 1092 & Adult 6 & Adult & 12785 & -19.7 & 9.1 & 3.2 & 12799 & -13.3 & -2.9 & 6.4 \\
\hline Feteira II & Fet 92 & Adult 7 & Adult & 12786 & -20.7 & 7.8 & 3.2 & 12800 & -12.0 & -2.7 & 8.7 \\
\hline Feteira II & Fet 1245 & Adult 8 & Adult & 12787 & -20.5 & 7.6 & 3.2 & 12801 & -11.1 & -2.6 & 9.4 \\
\hline Feteira II & Fet 968 & Adult 9 & Adult & 12788 & -20.1 & 8.3 & 3.2 & 12802 & & & \\
\hline Feteira II & Fet 799 & Adult 10 & Adult & 12789 & -20.3 & 8.2 & 3.2 & 12803 & -13.6 & -2.7 & 6.7 \\
\hline Feteira II & Fet 1229 & Adult 11 & Adult & 13808 & -20.9 & 7.6 & 3.3 & 13825 & -13.1 & -3.8 & 7.8 \\
\hline Feteira II & Fet 313 & Adult 12 & Adult & 13809 & -20.1 & 8.6 & 3.3 & 13826 & -13.7 & -3.3 & 6.4 \\
\hline Feteira II & Fet 1006 & Adult 13 & Adult & 13810 & -20.0 & 9.0 & 3.2 & 13827 & -13.7 & -2.7 & 6.3 \\
\hline Feteira II & Fet 561 & Juvenile 1 & $4-8 \mathrm{yr}$ & 12790 & -20.3 & 8.4 & 3.2 & 12804 & -11.7 & -2.0 & 8.6 \\
\hline Feteira II & Fet 567 & Juvenile 2 & $0-4 \mathrm{yr}$ & 12791 & -19.7 & 9.0 & 3.2 & 12805 & -14.0 & -3.3 & 5.7 \\
\hline Feteira II & Fet 316 & Juvenile 3 & $8-12 \mathrm{yr}$ & 12792 & -20.0 & 7.3 & 3.2 & 12806 & -13.2 & -3.3 & 6.8 \\
\hline Feteira II & Fet 749 & Juvenile 4 & $5-10 \mathrm{yr}$ & 12793 & -20.2 & 7.7 & 3.2 & 12807 & -13.8 & -2.5 & 6.4 \\
\hline Lapa da Rainha II & LR 261 & Adult 1 & Adult & 12808 & -19.6 & 8.6 & 3.2 & 12840 & -13.9 & -2.6 & 5.7 \\
\hline Lapa da Rainha II & LR 95 & Adult 2 & Adult & 12809 & -19.4 & 8.3 & 3.2 & 12841 & -13.3 & -2.9 & 6.1 \\
\hline Lapa da Rainha II & LR 47 & Adult 3 & Adult & 12810 & -19.7 & 9.8 & 3.2 & 12842 & -14.0 & -2.9 & 5.7 \\
\hline T. de Borracheira & Bor 1006 & Adult 1 & Adult & 12811 & & & & 12843 & -14.8 & -3.0 & \\
\hline T. de Borracheira & Bor 1007 & Adult 2 & Adult & 12812 & -19.7 & 8.9 & 3.2 & 12844 & -12.1 & -2.7 & 7.6 \\
\hline T. de Borracheira & Bor 57 & Adult 3 & Adult & 12813 & -19.8 & 8.6 & 3.2 & 12845 & -14.1 & -3.9 & 5.7 \\
\hline T. de Borracheira & Bor 61 & Juvenile 1 & $5-10 \mathrm{yr}$ & 12814 & -20.1 & 7.5 & 3.2 & 12846 & -15.0 & -2.9 & 5.1 \\
\hline T. de Borracheira & Bor 1000 & Juvenile 2 & $5-10 \mathrm{yr}$ & 12815 & -20.3 & 7.3 & 3.2 & 12847 & -11.9 & -2.8 & 8.4 \\
\hline Cova da Moura & CM 15 & Adult 1 & Adult & 12816 & & & & 12848 & -11.1 & -2.6 & \\
\hline Cova da Moura & CM 12 & Adult 2 & Adult & 12817 & -20.1 & 7.6 & 3.2 & 12849 & -13.3 & -2.7 & 6.8 \\
\hline Cova da Moura & $\mathrm{CM} 2$ & Adult 3 & Adult & 12818 & -19.3 & 9.7 & 3.2 & 12850 & -11.8 & -2.7 & 7.5 \\
\hline Cova da Moura & CM 84 & Adult 4 & Adult & 12819 & -19.5 & 9.5 & 3.2 & 12851 & -13.1 & -3.2 & 6.4 \\
\hline Cova da Moura & CM 159 & Adult 5 & Adult & 12820 & -19.5 & 9.9 & 3.2 & 12852 & -8.4 & -1.7 & 11.1 \\
\hline
\end{tabular}


Table 3 (Continued)

\begin{tabular}{|c|c|c|c|c|c|c|c|c|c|c|c|}
\hline Site & $\begin{array}{l}\text { Catalogue } \\
\text { number }\end{array}$ & Individual & $A g e^{*}$ & $\begin{array}{c}\text { USF laboratory } \\
\text { number }\end{array}$ & $\delta^{13} C_{c o}$ & $\delta^{15} N$ & $C: N$ & $\begin{array}{c}\text { USF laboratory } \\
\text { number }\end{array}$ & $\delta^{13} C_{a p}$ & $\delta^{18} O_{a p}$ & $\delta^{13} C_{c o-a p}$ \\
\hline Cova da Moura & CM 9 & Adult 6 & Adult & 12821 & -19.5 & 9.3 & 3.2 & 12853 & -10.0 & -2.5 & 9.5 \\
\hline Cova da Moura & CM 95 & Adult 7 & Adult & 12822 & -20.0 & 9.1 & 3.3 & 12854 & -9.7 & -2.8 & 10.3 \\
\hline Cova da Moura & CM 30 & Adult 8 & Adult & 12823 & -19.0 & 10.0 & 3.2 & 12855 & -12.8 & -3.2 & 6.2 \\
\hline Cova da Moura & CM 22 & Adult 9 & Adult & 12824 & -20.6 & 11.2 & 3.3 & 12856 & & & \\
\hline Cova da Moura & CM 81 & Adult 10 & Adult & 12825 & -20.5 & 8.7 & 3.3 & 12857 & -10.4 & -2.5 & 10.1 \\
\hline Cova da Moura & CM 43 & Juvenile 1 & $2-5 \mathrm{yr}$ & 12827 & -20.1 & 9.8 & 3.3 & 12859 & -8.4 & -1.3 & 11.7 \\
\hline Cova da Moura & CM 45 & Juvenile 2 & $5-10 \mathrm{yr}$ & 12828 & -20.2 & 8.4 & 3.3 & 12860 & -11.3 & -3.0 & 8.9 \\
\hline Cova da Moura & CM 16 & Juvenile 3 & $5-10 \mathrm{yr}$ & 12829 & -20.7 & 9.2 & 3.2 & 12861 & -11.9 & -2.5 & 8.8 \\
\hline Cova da Moura & CM 39 & Juvenile 4 & $10-20 \mathrm{yr}$ & 12826 & -21.0 & 9.3 & 3.3 & 12858 & -10.6 & -2.7 & 10.4 \\
\hline Bolores & 11.4.B14 & Adult 1 & Adult & $† 235488$ & -19.6 & 9.0 & & 13828 & -14.6 & -2.4 & 5.0 \\
\hline Bolores & 11N.4.B663 & Adult 2 & Adult & $† 249032$ & -19.5 & 9.5 & & 13829 & -12.9 & -3.5 & 6.6 \\
\hline Bolores & 9,10.0.BL5 & Adult 3 & Adult & 12833 & -20.8 & 9.8 & 3.4 & 12865 & -14.4 & -2.6 & 6.4 \\
\hline Bolores & 2.1.B31b & Adult 4 & Adult & $\dagger 256325$ & -19.7 & 9.6 & & 13830 & -15.1 & -2.0 & 4.4 \\
\hline Bolores & CR1.x.B89 & Adult 5 & Adult & 12830 & -20.4 & 8.0 & 3.3 & 12862 & -13.5 & -3.2 & 6.9 \\
\hline Bolores & CR2.x.B119 & Adult 6 & Adult & 12831 & & & & 12863 & -14.1 & -2.8 & \\
\hline Bolores & 4.1.B87 & Juvenile 1 & $0-3 \mathrm{yr}$ & 12834 & & & & 12866 & -14.3 & -2.3 & \\
\hline Bolores & x.x.B366a & Juvenile 2 & $18 \mathrm{~m}-3 \mathrm{yr}$ & 12835 & -20.8 & 8.9 & 3.3 & 12867 & -11.9 & -3.0 & 8.9 \\
\hline Bolores & 11.4.B56 & Juvenile 3 & $5-10 \mathrm{yr}$ & 12836 & -20.6 & 8.0 & 3.3 & 12868 & -12.7 & -2.9 & 7.9 \\
\hline Bolores & 4.1.B285 & Juvenile 4 & $5-10 \mathrm{yr}$ & 12837 & & & & 12869 & -14.8 & -3.1 & \\
\hline Bolores & H10B240 & Juvenile 5 & $5-10 \mathrm{yr}$ & $\dagger 235487$ & -20.4 & 7.5 & & & & & \\
\hline Bolores & 11S.4.B719 & Juvenile 6 & $14-16 \mathrm{yr}$ & 12832 & -20.4 & 8.6 & 3.2 & 12864 & & & \\
\hline \multirow[t]{6}{*}{ Bolores } & 11S.4.B819 & Juvenile 7 & $15-18 \mathrm{yr}$ & & & & & 13831 & -14.4 & -2.3 & \\
\hline & Average & & & & -20.1 & 8.7 & & & -12.9 & -2.6 & 7.3 \\
\hline & sd & & & & 0.5 & 0.9 & & & 1.6 & 0.6 & 1.7 \\
\hline & Low & & & & -21.3 & 7.3 & & & -15.1 & -3.9 & 4.4 \\
\hline & High & & & & -18.9 & 11.7 & & & -6.1 & -0.8 & 13.9 \\
\hline & Range & & & & 2.4 & 4.4 & & & 9.0 & 3.1 & 9.5 \\
\hline
\end{tabular}

*m, Months; yr, years.

$\uparrow$ Collagen data from Beta Analytic. 


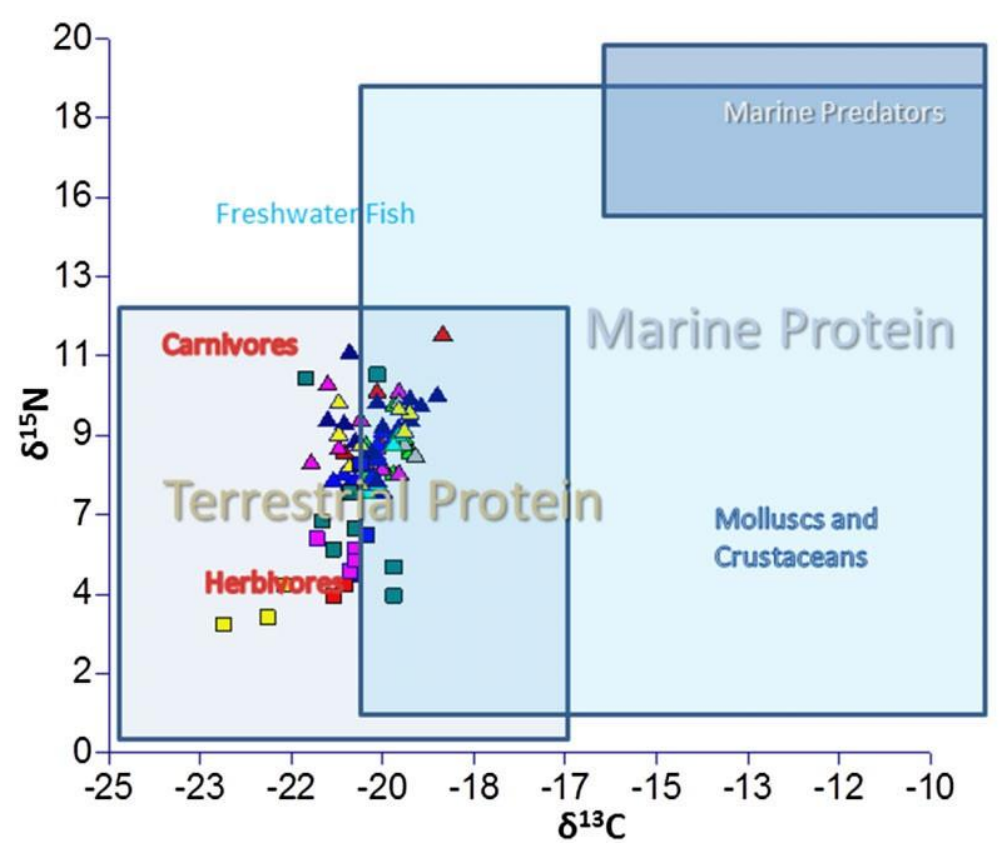

$\triangle$ Cabeca da Arrudal

$\Delta$ Zambujal

$\Delta$ Paimogol

- Feteirall

$\Delta$ Lapa da Rainha II

$\triangle$ T. de Borrachera

- Cova da Moura

$\triangle$ Bolores

- Bos

a Sus

- Cervus

- Ovis/Capra

口 Orycdagus

Figure 2 Scatter plots of collagen values for humans and fauna.

statistically significant differences were present for $\delta^{15} \mathrm{~N}$ by age-grade $(p=0.008024)$. Using the Mann-Whitney $U$ test, the statistically significant differences were found to occur only between adults and older juveniles ( $p=0.00188)$.

DISCUSSION

\section{Fauna}

Based on the collagen data, the sampled fauna obtained the majority of their protein from a food web based upon $\mathrm{C}_{3}$ plants and, in the case of pigs, terrestrial animal proteins. Overall, the animals exhibit low standard deviations in $\delta^{13} \mathrm{C}_{\mathrm{co}}$ values $( \pm 0.01-0.06)$ within each species and, with the exception of rabbits, overlap in range. Variations in $\delta^{13} \mathrm{C}_{\mathrm{co}}$ values between rabbits are probably due to limited home ranges. The larger variation in the $\delta^{13} \mathrm{C}_{\mathrm{co}}$ values for the ovicaprids suggests the inclusion of goats in the sample, as goats are browsers and may obtain protein from broadleaf plants, shrubs and other non-grasses. Pigs, the only sampled omnivores, have the largest $\delta^{13} \mathrm{C}_{\mathrm{co}}$ range. The faunal $\delta^{13} \mathrm{C}_{\mathrm{ap}}$ values were more varied than the $\delta^{13} \mathrm{C}_{\mathrm{co}}$ values, with especially high standard deviations recorded for the pigs, ovicaprids and rabbits. Many of the animals exhibited slightly enriched $\delta^{13} \mathrm{C}_{\mathrm{ap}}$ values that may indicate the consumption of some $\mathrm{C}_{4}$ plants. Few $\mathrm{C}_{4}$ plants are known to have existed at this location during the LN/CA, but possible sources could include seaweed and sea grasses from the large estuarine environment that existed during this time (Kunst 1995).

As expected, depleted $\delta^{15} \mathrm{~N}$ values were obtained for most herbivores, as these animals occupy lower trophic levels in the food chain. Data from three cows are the exception. These higher $\delta^{15} \mathrm{~N}$ 


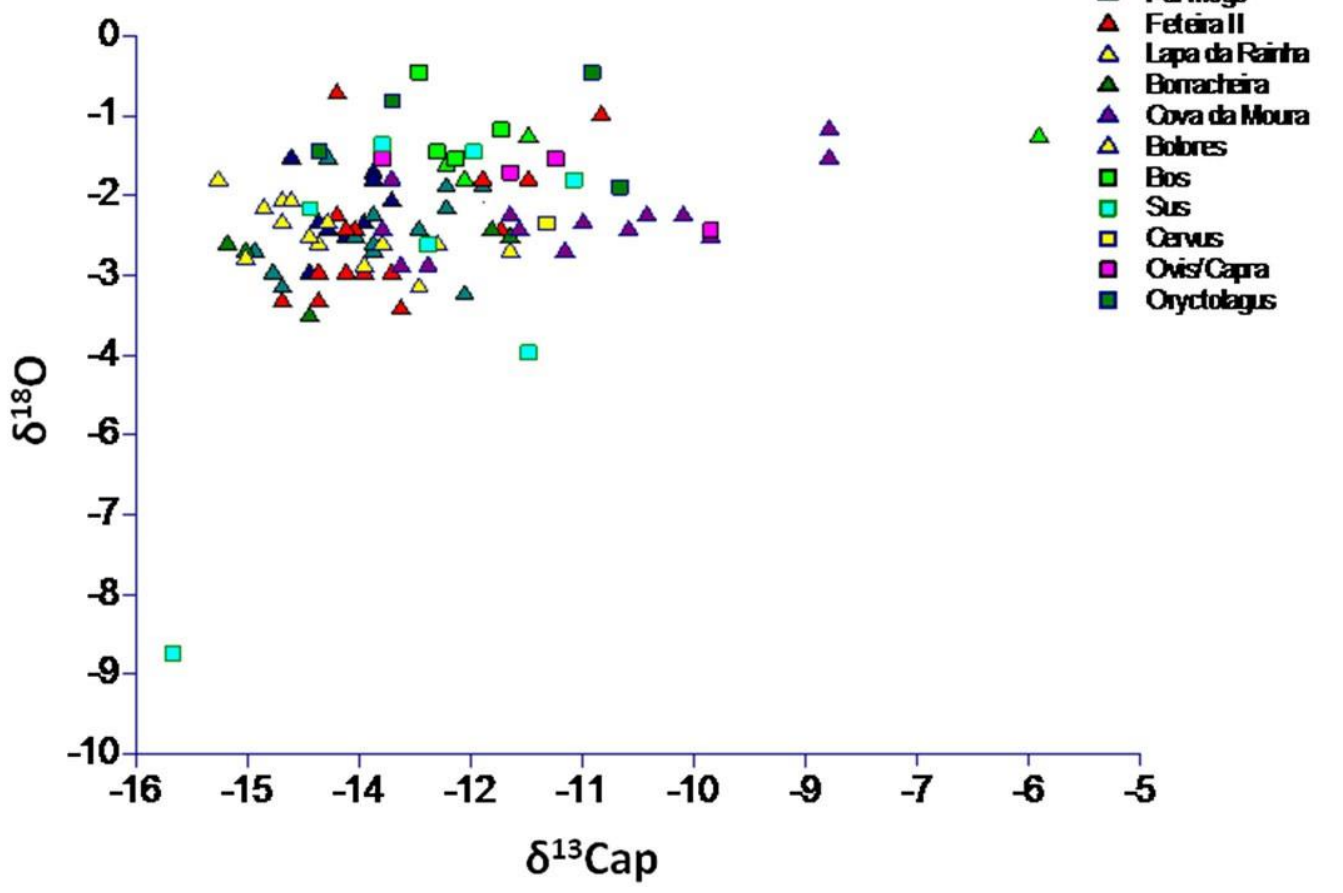

Figure 3 Scatter plots of apatite values for humans and fauna.

values probably point to the consumption of a nitrogen-enriched fodder. Another possibility is that the cows grazed on nitrogen-enriched saltwater marsh plants, as elevated $\delta^{15} \mathrm{~N}$ values have been documented in animals grazing in saltwater marsh environments in other prehistoric settings (Britton et al. 2008). The deer and rabbits exhibit the lowest $\delta^{15} \mathrm{~N}$ values, reflecting a diet based on low-protein wild plants. The more varied $\delta^{15} \mathrm{~N}$ values for the ovicaprids, again, probably reflect the more diverse feeding patterns of goats. Pigs exhibit the most enrichment, and the largest range, in $\delta^{15} \mathrm{~N}$ values. This suggests that some pigs were probably being fed human food waste containing terrestrial animal proteins. Pigs also exhibit the highest variation in the $\delta^{18} \mathrm{O}$ values. As $\delta^{18} \mathrm{O}$ values reflect water intake, this variation may be attributed to pigs' larger dietary range and the likelihood that they accessed more diverse water sources. Strongly depleted $\delta^{18} \mathrm{O}$ levels were recorded for two pigs, which may indicate that both were moved or traded into this area.

\section{Human populations}

The human collagen data also indicate diets composed of mainly terrestrial animal proteins and $\mathrm{C}_{3}$ plants. Although a few individuals exhibit enriched $\delta^{13} \mathrm{C}_{\mathrm{co}}$ and $\delta{ }^{15} \mathrm{~N}$ values that may indicate some marine protein input, there is little evidence that marine protein was a dietary staple in these communities, despite living in an estuary environment close to the sea. This dietary signature is strongly divergent from those of earlier Mesolithic populations, for which isotopic analyses show a strong reliance on marine proteins (Lubell et al. 1994; Carvalho and Petchey 2013). While the 
relative lack of marine proteins may seem counterintuitive in this estuarine environment, a strong dependence on terrestrial proteins in Neolithic populations, regardless of the accessibility of marine resources, is a trend seen in many parts of Europe (Tauber 1983; Schulting and Richards 2001) and is even noted in many island contexts, such as Sardinia (Lai et al. 2007) and the Balearic Islands (Davis 2002; Van Strydonck et al. 2002). It has been argued that this may reflect ideological changes related to food valuation, in addition to the increased availability of terrestrial protein sources through the domestication of animals (Thomas 2003). However, fishing implements and fish and shellfish remains recovered from settlement and burial sites (Cardoso 2007) suggest that some marine proteins were being consumed. Milner et al. (2004) contend that, due to physiological processes related to isotope fractionation and collagen formation, individuals may consume up to $20 \%$ of all protein from carbon-enriched marine foods without exhibiting an enriched $\delta^{13} \mathrm{C}_{\text {co }}$ signature. Therefore, it is likely that marine proteins continued to be of some value. Considering the pairing of enriched $\delta^{13} \mathrm{C}_{\text {co }}$ and $\delta^{15} \mathrm{~N}$ values in several of the sampled individuals, some marine dietary input is indeed evident.

The $\delta^{15} \mathrm{~N}$ values were more variable than the $\delta^{13} \mathrm{C}_{\mathrm{co}}$, with standard deviations for most burials ranging between 0.6 and $0.9 \%$. The exception is the only settlement in the study, Zambujal, but this greater diversity can be attributed to the small sample size and a neonate with extremely elevated $\delta^{15} \mathrm{~N}$ values - probably related to in utero skeletal development and consumption of breast milk. In the other burials, some of the variation in $\delta^{15} \mathrm{~N}$ values may also be attributable to age-related differences. In general, for juveniles, $\delta^{15} \mathrm{~N}$ values commonly fall in the low or high ranges when compared to adults. The ones with the highest values tend to be the youngest in the sample, and these values are most probably a product of breast milk consumption. However, with the exception of Juvenile 1 from Cova da Moura, most young juveniles do not exhibit enriched $\delta^{18} \mathrm{O}$ or $\delta^{13} \mathrm{C}$ values, which suggests that supplemental water and carbohydrates were being introduced in late infancy. The $\delta^{15} \mathrm{~N}$ values appear to decline as the juveniles in the sample move into early childhood, probably reflecting the cessation of breast milk consumption and ongoing bone remodelling related to skeletal growth. Older juveniles, with age-at-death ranges between 5 and 10 years of age, exhibit statistically significant differences in $\delta^{15} \mathrm{~N}$ values when compared to adults. These low $\delta^{15} \mathrm{~N}$ values for older juveniles may indicate culturally mediated differences in adult and child diets (i.e., that children consumed less or different types of protein than adults). Although health status was, in general, good in this region, based upon skeletal indicators of pathology (Silva 2003; Waterman 2012), demographic patterns in the surveyed burials suggest that child mortality was high (Waterman and Thomas 2011). Insufficient protein intake could have exacerbated other health-related issues and contributed to the high mortality rates for juveniles. However, it is also possible that the lower $\delta^{15} \mathrm{~N}$ values are caused by physiological differences related to bone growth and development. Delayed growth has been shown to result in the isotopic enrichment of hard tissues (Warinner and Tuross 2010). Based upon this finding, we may expect that rapid skeletal growth during childhood may lead to isotope depletion. Nevertheless, Waters-Rist and Katzenberg (2010) found no evidence of differences in $\delta^{15} \mathrm{~N}$ values in juvenile long bones from a protohistoric Canadian ossuary. Thus, the effect of growth on $\delta^{15} \mathrm{~N}$ values is unclear. A final consideration is that the juveniles in these burials did not reach adulthood. Thus, this sample is probably dominated by juveniles with already compromised health statuses. Therefore, these lower $\delta^{15} \mathrm{~N}$ values may reflect the fact that juveniles who consumed less protein had a higher mortality risk, rather than a sign of generalized culturally mediated dietary differences according to age.

While age-at-death may account for some of the isotopic variation, it cannot account for all intra-group variations in protein consumption, as in some burials the highest and lowest $\delta^{15} \mathrm{~N}$ 
values were found in adults (e.g., Cova da Moura Adults 2 and 9). At their most extreme, these disparities represent a full trophic level (2-3\%) and suggest that some protein-related differentiation in adult diet occurred within burial populations. In some instances, when there is corresponding enrichment in $\delta{ }^{13} \mathrm{C}_{\mathrm{co}}$ values, the higher $\delta^{15} \mathrm{~N}$ values suggest marine protein consumption (such as Adult 6 from Feteira II, or Adult 8 from Cova da Moura). While most burials exhibit some internal variation in $\delta^{15} \mathrm{~N}$ values, only Cova da Moura and Zambujal appear to diverge from the other groups, with average values of 9.4\%o and 9.7\%o. While the Zambujal differences can be attributed to the neonate outlier and the small sample size, for Cova da Moura these differences can only be explained by differential protein intake - either in the amount or type consumed. In the case of Cova da Moura, statistically significant differences in $\delta^{15} \mathrm{~N}$ values were found between Cova da Moura and Feteira II and Cova da Moura and Paimogo I. Thus, it appears that there is evidence of dietary differentiation in protein intake by burial site.

The human $\delta^{13} C_{\text {ap }}$ data were also highly variable, with some individuals exhibiting significant enrichment in their $\delta^{13} \mathrm{C}_{\mathrm{ap}}$ values. As this enrichment is only strongly exhibited in the apatite, these values may best be attributable to the consumption of $\left(\mathrm{C}_{3}\right.$-based $)$ terrestrial proteins and $\mathrm{C}_{4}$ plants, with the plants contributing mainly to the carbohydrate or fat component of the diet (Ambrose et al. 1997). As mentioned previously, few terrestrial $\mathrm{C}_{4}$ plants are native to Portugal, and most are grasses and sedges that are inedible for humans. The $\mathrm{C}_{4}$ plant millet (Panicum miliaceum) is known to have been in Spain by the Middle Bronze Age (Rovira Buendía 2007), and it is possible that the enriched $\delta^{13} \mathrm{C}_{\mathrm{ap}}$ values suggest its early introduction into Portugal. However, unlike other early introduction sites for millet in Europe (Tafuri et al. 2009), here there is no evidence for the large-scale use of millet as a human food or animal fodder. If millet did arrive earlier than is commonly thought, then it may have been grown and used for specialpurpose foods - such as millet beer-rather than as a staple crop. Alternative possibilities are that some humans consumed seaweed ( $\mathrm{a}_{4}$ plant). Seaweed is also an excellent fertilizer and has been used to fertilize crops throughout costal Portugal for hundreds of years. It is possible that during the LN/CA this practice had already been adopted and that the use of seaweed as a fertilizer led to $\delta^{13} \mathrm{C}$ enrichment of $\mathrm{C}_{3}$ plants by increasing the $\delta^{13} \mathrm{C}$ values of the soil and by increasing the soil salinity (Farquhar et al. 1989). This enrichment, in turn, may have been passed on to the plant consumers. Enriched $\delta^{13} \mathrm{C}_{\text {ap }}$ values could also be attributed to possible bone diagenesis, although precautions were taken during sample preparation and processing to remove non-biogenic carbonates.

The $\delta^{13} \mathrm{C}_{\text {ap }}$ values vary strongly between burial populations, probably reflecting differences in plant intake. Individuals from Cova da Moura, Feteira II and Zambujal all exhibit enriched $\delta^{13} \mathrm{C}_{\mathrm{ap}}$ values that suggest $\mathrm{C}_{4}$ plant consumption. Statistically significant differences in $\delta^{13} \mathrm{C}_{\text {ap }}$ values were found between Cova da Moura and four other sites (Paimogo I, Feteira II, Cabeço da Arruda I and Bolores) and between Bolores and Feteira II.

Humans had more homogeneous $\delta^{18} \mathrm{O}$ values than the sampled pigs, perhaps signifying that in general humans consumed water from similar sources and environments. With the exception of Zambujal, the majority of sites have similar $\delta^{18} \mathrm{O}$ averages $\left(-2.4\right.$ to $-2.8 \%$ ). Based upon $\delta^{18} \mathrm{O}$ values, no statistically significant differences between burial sites were recognized and no migrants were identified. In some cases, such as Juvenile 1 from Cova da Moura, enriched oxygen isotopes probably reflect breast milk consumption.

Based on an evaluation of the $\delta^{13} \mathrm{C}_{\mathrm{co}}$ and $\delta^{13} \mathrm{C}_{\mathrm{ap}}$ spacing in the combined faunal and human data, using the Kellner and Schoeninger (2007) model of dietary inputs (Fig. 4), it is clear that the LN/CA humans cluster tightly around the $\mathrm{C}_{3}$ protein line. The dietary energy (carbohydrate) sources, as reflected in the $\delta^{13} \mathrm{C}_{\mathrm{ap}}$ values, are more diverse for both the humans and sampled 


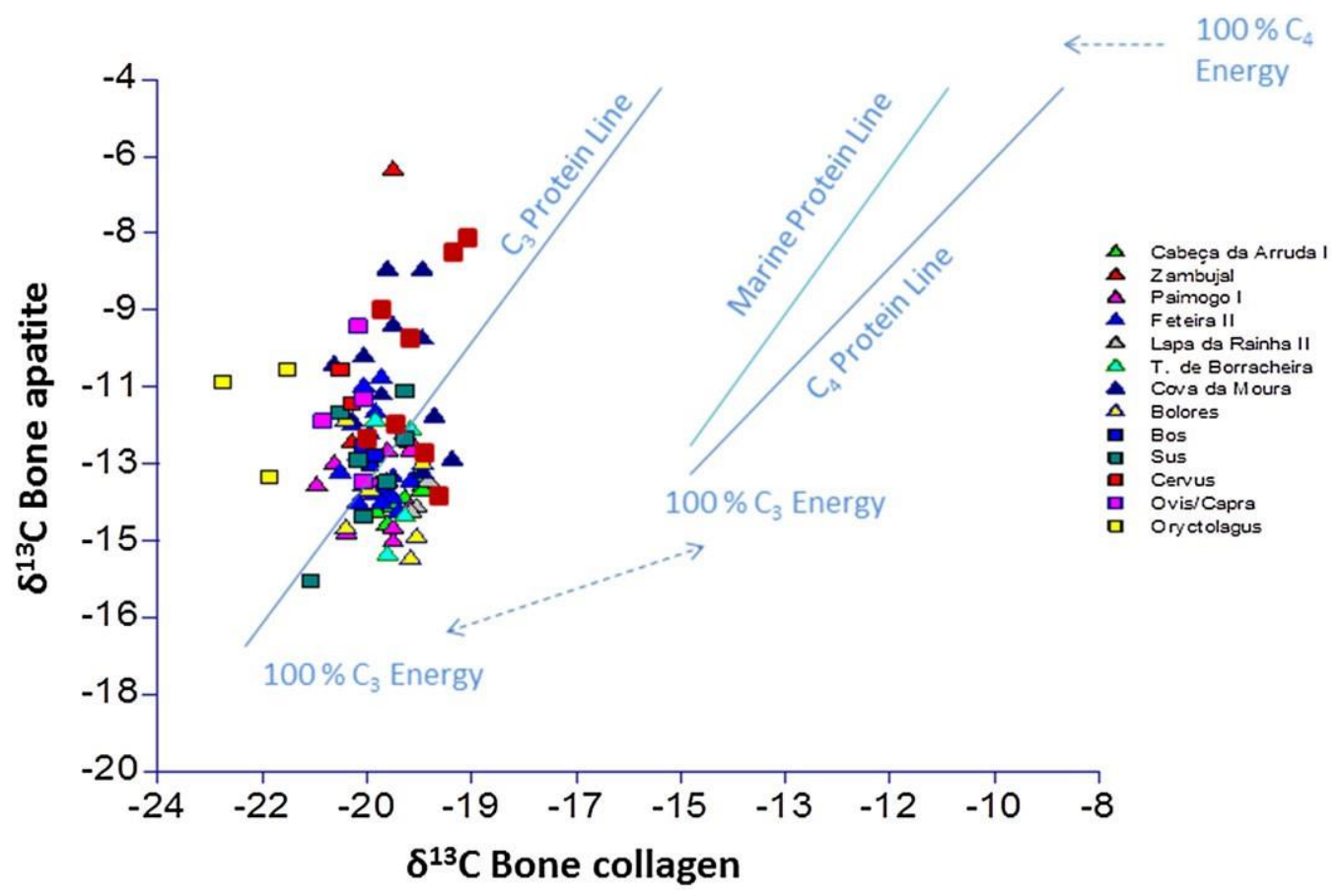

Figure 4 A scatter plot comparing $\delta^{13} C_{c o}$ and $\delta^{13} C_{a p}$ values for humans by burial site and fauna, using the linear dietary model for carbon isotopes proposed by Kellner and Schoeninger (2007).

animals. However, within the human population the $\delta^{13} \mathrm{C}_{\text {ap }}$ enrichment is stronger - marking clear outliers who must have received some portion of their dietary energy in the form of $\mathrm{C}_{4}$ plants. When burials are compared, it is clear that Cova da Moura's $\delta^{13} \mathrm{C}_{\mathrm{ap}}$ values are divergent from those of the other sites. Only Zambujal has an individual whose $\delta^{13} \mathrm{C}_{\text {ap }}$ value exceeds those of Cova da Moura (Juvenile 2).

\section{Diet and social differentiation}

The goal of this research was to ascertain if there are discernible differences in biological markers of diet in individuals interred at seven geographically close LN/CA burials that could be used to distinguish socially differentiated populations. Statistically significant differences in more than one variable were found between several sites. The most pronounced differences were found between individuals interred at Cova da Moura and those interred at the other selected sites. These results suggest two main dietary differences at Cova da Moura. One is that individuals appear to have eaten more animal protein in general or, perhaps, relied more heavily on the protein of nitrogen-enriched animals. Faunal analyses have documented large numbers of pig remains at Zambujal (Harrison 1985; Albarella et al. 2005) and the analyses presented here document that some of these pigs were eating a significant amount of animal protein. If the Cova da Moura individuals were consuming pigs that were eating large amounts of meat, this could account for the more enriched $\delta^{15} \mathrm{~N}$ values. Alternatively, the people buried at Cova da Moura may have simply been eating substantially more protein. In many societies, meat (or particular types of meat) is considered a status food and its consumption can have important social 
applications, such as in ritual feasting (Wiessner and Schiefenhovel 1996). In the case of Cova da Moura, the enriched $\delta^{15} \mathrm{~N}$ values may be attributed to status-related meat consumption; however, without a more nuanced understanding of food production and politics during this time, it is impossible to say how the differential protein intake was related to social organization.

The second significant dietary difference found at Cova da Moura is that individuals appear to have been consuming $\mathrm{C}_{4}$ plants. Although some $\delta^{13} \mathrm{C}_{\mathrm{ap}}$ enrichment could be tied to marine animal consumption, based on the Kellner and Schoeninger (2007) model, the lack of enrichment in collagen suggests that it was from a largely carbohydrate source. While individuals from Cova da Moura exhibit more enriched $\delta^{13} \mathrm{C}_{\mathrm{ap}}$ values than individuals from most other sites, there is evidence of some $\mathrm{C}_{4}$ plant intake at Zambujal and, to a lesser extent, Feteira II. Thus, we see that these differences are not completely confined to Cova da Moura. Using strontium isotope analysis on humans interred within these same burials, Waterman et al. (2014b) found that $4 / 5$ identified migrants were from Cova da Moura. Of these Cova da Moura migrants, $3 / 4$ exhibit enriched $\delta^{13} \mathrm{C}_{\mathrm{ap}}$ values, suggesting that the dietary differences at this site may relate to non-local dietary practices or non-native foods. However, 'local' individuals from Cova da Moura also exhibit enriched $\delta^{13} \mathrm{C}_{\mathrm{ap}}$ and $\delta^{15} \mathrm{~N}$ values; thus we cannot tie these differences purely to migratory status.

The two burials with the strongest evidence of intake of $\mathrm{C}_{4}$ plants, Cova da Moura and Feteira II, have the earliest inception dates. If the $\delta^{13} \mathrm{C}_{\mathrm{ap}}$ values are evidence of millet's early introduction to the region, it seems counterintuitive that these values are present in the oldest burials and absent from the youngest. Beginning in the Mesolithic, the estuary in the region began to slowly fill in (Lord et al. 2010). If the enriched $\delta^{13} \mathrm{C}_{\text {ap }}$ values are attributable to a native estuary plant, its absence from the diet of later populations may be explained by environmental degradation forcing changes in food production and consumption. In support of this idea, Cova da Moura and Feteira II both have statistically significantly enriched $\delta^{13} C_{a p}$ values when compared to Bolores, the youngest burial site in this survey. However, radiocarbon dates for both Cova da Moura and Feteira II reveal long use-lives (Table 1); therefore, the exact diachronic sequences of the sampled individuals are unclear.

\section{CONCLUSION}

The goal of this study was to discern if significant dietary differences exist between people interred in seven LN/CA Portuguese collective burials that could provide information about social differentiation. Based upon the stable isotopic data, people from all burials subsisted mainly on terrestrial animal proteins and $\mathrm{C}_{3}$ plants, with limited marine protein input. The consistency of this data across the surveyed sites suggests regional dietary stability and integration throughout the Neolithic and the Copper Age.

However, older juveniles exhibited statistically significant differences in $\delta^{15} \mathrm{~N}$ values when compared to adults. These lower $\delta^{15} \mathrm{~N}$ values for older juveniles may indicate culturally mediated differences in adult and child diets, or may relate to aspects of bone growth and development. For juveniles in the Estremadura during the LN/CA, insufficient protein intake may have exacerbated other health-related issues and contributed to the high childhood mortality rates. The apatite data suggest dietary differentiation in plant consumption, and some humans and animals exhibited enriched $\delta^{13} C_{a p}$ values that may be attributable to $C_{4}$ plant intake. This finding may indicate the early introduction of millet into Portugal, the consumption of seaweed and its possible use as a fertilizer, or the consumption of unknown native $\mathrm{C}_{4}$ plants.

Statistically significant differences in protein and/or plant intake were found between Cova da Moura and four of the other burials. Additionally, statistically significant differences in plant 
intake were found between Bolores and Feteira II. At sites with sample sizes that were too small for statistical analyses, divergent dietary trends were also noted. Thus, we proposed that biological evidence of social differentiation can be obtained from complicated commingled burial assemblages - such as those found in the Estremadura - using the employed methodology. The question that remains, however, is as follows: how are these findings related to patterns of social organization in the region during the Late Neolithic and the Copper Age? We suggest that while dietary differentiation alone is not adequate evidence of high or low social status, when these data are combined with other evidence, it may be possible for larger patterns to emerge. For example, there is clear evidence of both dietary differentiation and migrant individuals at Cova da Moura. There are also a higher number and greater diversity of rare raw materials and well-crafted beads and pendants obtained via long-distance exchange in the burials at Cova da Moura (Thomas 2011). Thus, we can begin to see a pattern of social differentiation in the Cova da Moura burials that marks the individuals interred there as special in terms of diet, mobility and perhaps personal ornaments or burial garments. However, with its long use-life, a more precise chronology of the individuals interred at Cova da Moura is needed to clarify these results. Additional work that focuses on increasing the available biological data sets from the Late Neolithic - Early Bronze Age collective burials of the Iberian Peninsula will provide us with a more nuanced understanding of life and death during the rise — and fall—of early complex societies.

\section{ACKNOWLEDGEMENTS}

We would like to thank Jonathan Thomas and Katina Lillios of the University of Iowa, and Isabel Luna of the Museu Municipal Leonel Trindade and the Centro de Investigação em Antropologia e Saúde (CIAS), for help and support with this project. Funds for this project were provided by the Archaeological Institute of America Archaeology of Portugal Fellowship and the University of Iowa T. Anne Cleary Dissertation Fellowship.

\section{REFERENCES}

Albarella, U., Davis, S. J. M., Detry, C., and Rowley-Conwy, P. A., 2005, Pigs of the 'Far West': the biometry of Sus from archaeological sites in Portugal, Anthropozoologica, 40, 27-54.

Ambrose, S. H., and Krigbaum, J., 2003, Bone chemistry and bioarchaeology, Journal of Anthropological Archaeology, 22, 193-9.

Ambrose, S. H., and Norr, L., 1993, Experimental evidence for the relationship of the carbon isotope ratios of whole diet and dietary protein to those of bone collagen and carbonate, in Prehistoric human bone: archaeology at the molecular level (eds. J. B. Lambert and G. Grupe), 1-37, Springer-Verlag, Berlin.

Ambrose, S. H., Butler, B. M., Hanson, D. B., Hunter-Anderson, R. L., and Krueger, H. W., 1997, Stable isotopic analysis of human diet in the Marianas Archipelago, Western Pacific, American Journal of Physical Anthropology, 104(3), 343-61.

Bentley, R. A., 2006, Strontium isotopes from the earth to the archaeological skeleton: a review, Journal of Archaeological Method and Theory, 13(3), 135-87.

Britton, K., Müldner, G., and Bell, M., 2008, Stable isotope evidence for salt-marsh grazing in the Bronze Age Severn Estuary, UK: implications for palaeodietary analysis at coastal sites, Journal of Archaeological Science, 35(8), 211118.

Cardoso, J. L., 2007, Pré-história de Portugal, Universidade Aberta, Lisboa.

Carvalho, A., and Petchey, F., 2013, Stable isotope evidence of Neolithic palaeodiets in the coastal regions of southern Portugal, Journal of Island \& Coastal Archaeology, 8, 361-83.

Chapman, R., 2003, Archaeologies of complexity, 166-75, Routledge, London.

Chapman, R., 2008, Producing inequalities: regional sequences in later prehistoric southern Spain, Journal of World Prehistory, 21, 195-260. 
Chisholm, B. S., Nelson, D. E., and Schwarcz, H. P., 1982, Stable-carbon isotope ratios as a measure of marine versus terrestrial protein in ancient diets, Science, 216(4550), 1131-2.

Cucina, A., and I s, can, M. Y., 1997, Assessment of enamel hypoplasia in a high status burial site, American Journal of Human Biology, 9(1), 213-22.

Cunha, E., Umbelino, C., Silva, A. M., and Cardoso, F., 2007, What can pathology say about the Mesolithic and Late Neolithic/Chalcolithic communities? The Portuguese case, in Ancient health: skeletal indicators of agricultural and economic intensification (eds. M. Cohen, M. Gillian and M. Crane-Kramer), 164-75, University Press of Florida, Gainesville, FL.

Davis, M. H. L. A., 2002, Putting meat on the bone: an investigation into palaeodiet in the Balearic Islands using carbon and nitrogen stable isotope analysis, in World islands in prehistory: international insular investigations, proceedings of the V Deia International Conference of Prehistory (eds. W. H. Waldren and J. A. Ensenyat), 198-213, BAR International Series, 1095, Archaeopress, Oxford.

DeNiro, M. J., 1985, Postmortem preservation and alteration of in vivo bone collagen isotope ratios in relation to paleodietary reconstruction, Nature, 317, 806-9.

Díaz-del-Río, P., 2011, Labor in the making of Copper Age lineages in Iberia, in Comparative archaeologies: prehistoric Iberia (3000-1500 BC) and the American Southwest (AD 900-1600) (ed. K. T. Lillios), 37-56, Oxbow, Oxford.

Farmer, P., 2003, Infections and inequalities: the modern plagues, University of California Press, Berkeley, CA.

Farquhar, G. D., Ehleringer, J. R., and Hubick, K. T., 1989, Carbon isotope discrimination and photosynthesis, Annual Review of Plant Biology, 40(1), 503-37.

Fogel, M., Tuross, N., and Owsley, D. W., 1989, Nitrogen isotope tracers, Carnegie Institute Yearbook, 88, $133-4$.

Forenbaher, S., 1999, Production and exchange of bifacial flaked stone artifacts during the Portuguese Chalcolithic, Archaeopress, Oxford.

Fuller, B. T., Fuller, J. L., Harris, D. A., and Hedges, R. E. M., 2006, Detection of breastfeeding and weaning in modern human infants with carbon and nitrogen stable isotope ratios, American Journal of Physical Anthropology, 129(2), 279-93.

Gallay, G., Spindler, K., Trindade L., and da Veiga Ferreira, O., 1973, O monumento pré-histórico de Pai Mogo (Lourinhã), Associação dos Arqueólogos Portugueses, Lisbon.

Gonçalves, V. S., 1994, A primeira metade do $3^{\circ}$ milenio no Centro/Sul de Portugal: algumas breves reflexôes, enquanto outras nâo são posiveis, Trabalhos de Arqueologia e Etnologia, 34(3), 117-31.

Gonçalves, V.S., 1999, Time, landscape and burials 1. Megalithic rites of ancient peasant societies in central and southern Portugal: an initial review, Journal of Iberian Archaeology, 1, 83-109.

Harrison, R. J., 1985, The 'policultivo ganadero', or the secondary products revolution in Spanish agriculture, 50001000 bc, Proceedings of the Prehistoric Society, 51, 75-102.

Horwath, B., Waterman, A. J., Lillios, K. T., and Irish, J., 2014, Assessing change in diet and biological affinity between the 4th and 3rd millennia bce in the Portuguese Estremadura through microwear and dental morphology: a preliminary comparison of Feteira II and Bolores, Homo-Journal of Comparative Human Biology, 65(2), 87-100.

Jorge, V. O., 1995, Late prehistoric funerary mounds in northern Portugal as indicators of social complexity, in The origins of complex societies in late prehistoric Iberia (ed. K. T. Lillios), 140-52, International Monographs in Prehistory, Ann Arbor, MI.

Katzenberg, M. A., 2008, Stable isotope analysis: a tool for studying past diet, demography, and life history, in Biological anthropology of the human skeleton, 2nd edn (eds. M. A. Katzenberg and S. R. Saunders), 413-41, John Wiley and Sons, Inc., Hoboken, NJ.

Katzenberg, M. A., Saunders, S. R., and Fitzgerald, W. R., 1993, Age-differences in stable carbon and nitrogen isotope ratios in a population of prehistoric maize horticulturists, American Journal of Physical Anthropology, 90(3), $267-81$.

Kellner, C. M., and Schoeninger, M. J., 2007, A simple carbon isotope model for reconstructing prehistoric human diet, American Journal of Physical Anthropology, 133(4), 1112-27.

Koch, P. L., Tuross, N., and Fogel, M. L., 1997, The effects of sample treatment and diagenesis on the isotopic integrity of carbonate in biogenic hydroxylapatite, Journal of Archaeological Science, 24, 417-29.

Krueger, H. W., and Sullivan, C. H., 1984, Models for carbon isotope fractionation between diet and bone, in Stable isotopes in nutrition (eds. J. Turnlund and P. E. Johnson), 205-22, American Chemical Society, Washington, DC.

Kunst, M., 1995, Central places and social complexity in the Iberian Copper Age, in The origins of complex societies in late prehistoric Iberia (ed. K. T. Lillios), 32-43, International Monographs in Prehistory, Ann Arbor, MI.

Kunst, M., and Lutz, N., 2008, Zambujal (Torres Vedras, Portugal). Zur Präzision der absoluten chronologie durch die untersuchungen an der vierten befestigungslinie, Madrider Mitteilungen, 49, 29-62.

Kunst, M., and Trindade, L., 1990, Zur besiedlungesgeschicte des Sizandrotals: Ergebnisse aus der küstenforschung, Madrider Mitteilungen, 31, 34-83. 
Kunst, M., Cardoso, J. L., and Waterman, A. J., 2012, Human bones from Chalcolithic walled enclosures of Portuguese Estremadura: examples of Zambujal and Leceia, Paper presented at the International Meeting of Recent Pre-historic

Enclosures and Funerary Practices (organized by A. Valera and ERA-Arqueologia), Lisboa, Portugal, 6-8 November. Lai, L., Tykot, R. H., Beckett, J. F., Floris, R., Fonzo, O., Usai, E., Manunza, M. R., Goddard, E., and Hollander, D., 2007, Interpreting stable isotopic analyses: case studies on Sardinian prehistory, in Archaeological chemistry: analytical techniques and archaeological interpretation (eds. M. D. Glascock, R. J. Speakman and R. S. Popelka-Filcoff), 114-36, ACS Symposium Series 968, American Chemical Society, Washington, DC.

Le Bras-Goude, G., Herrscher, E., and Vaquer, J., 2013, Funeral practices and foodstuff behaviour: What does eat meat mean? Stable isotope analysis of Middle Neolithic populations in the Languedoc region (France), Journal of Anthropological Archaeology, 32(3), 280-7.

Le Huray, J. D., and Schutkowski, H., 2005, Diet and social status during the La Tene period in Bohemia: carbon and nitrogen stable isotope analysis of bone collagen from Kutna Hora-Karlov and Radovesice, Journal of Anthropological Archaeology, 24(2), 135-47.

Lee-Thorp, J. A., Sealy, J. C., and van der Merwe, N. J., 1989, Stable carbon isotope ratio differences between bonecollagen and bone apatite, and their relationship to diet, Journal of Archaeological Science, 16, 585-99.

Leisner, V., 1965, Die megalithgräber der Iberischen Halbinsel. Der Westen, Walter de Gruyter, Berlin.

Lillios, K. T., 1993, Regional settlement abandonment at the end of the Copper Age in the lowlands of west-central Portugal, in Abandonment of settlements and regions: ethno-archaeological and archaeological approaches (eds. C. Cameron and S. Tomka), 110-20, Cambridge University Press, Cambridge.

Lillios, K. T., Waterman, A. J., Artz, J., and Josephs, R., 2010, The Neolithic - Early Bronze Age mortuary rockshelter of Bolores, Torres Vedras, Portugal. Preliminary results on the 2007 and 2008 excavations, Journal of Field Archaeology, 35(1), 19-39.

Lord, A., Cabral, M., C., Dambeck, R., and Kunst, M., 2010, Ostracod evidence for the Neolithic environment of Rio Sizandro, Portugal, Palaeobiodiversity and Palaeoenvironments, 91(3), 1-14.

Lubell, D., Jackes, M., Schwarcz, H., Knyf, M., and Meiklejohn, C., 1994, The Mesolithic-Neolithic transition in Portugal: isotopic and dental evidence of diet, Journal of Archaeological Science, 21(1), 201-16.

Manolagas, S. C., 2000, Birth and death of bone cells: basic regulatory mechanisms and implications for the pathogenesis and treatment of osteoporosis, Endocrine Reviews, 21(2), 115-37.

Milner, N., Craig, O. E., Bailey, G. N., Pedersen, K., and Andersen, S. H., 2004, Something fishy in the Neolithic? A reevaluation of stable isotope analysis of Mesolithic and Neolithic coastal populations, Antiquity, 78(299), 9-22.

Montgomery, J., Evans, J. A., Powlesland, D., and Roberts, C. A., 2005, Continuity or colonization in Anglo-Saxon England? Isotope evidence for mobility, subsistence practice and status at West Heslerton, American Journal of Physical Anthropology, 126, 123-38.

Norr, L., 1995, Interpreting dietary maize from bone stable isotopes in the American tropics: the state of the art, in Archaeology in the lowland American tropics: current analytical methods and applications (ed. P. W. Stahl), 198-223, Cambridge University Press, Cambridge.

Owsley, D. W., Orser, C. E., Mann, R. W., Moore-Jansen, P. H., and Montgomery, R. L., 1987, Demography and pathology of an urban slave population from New Orleans, American Journal of Physical Anthropology, 74(2), 18597.

Paine, R. R., Vargiu, R., Coppa, A., Morselli, C., and Schneider, E. E., 2007, A health assessment of high status Christian burials recovered from the Roman-Byzantine archeological site of Elaiussa Sebaste, Turkey, Homo-Journal of Comparative Human Biology, 58(2), 173-90.

Powell, M. L., 1992, Health and disease in the late prehistoric Southeast, in Disease and demography in the Americas (eds. J. W. Verano and D. H. Ubelaker), 41-53, Smithsonian Institution Press, Washington, DC.

Price, T. D., Wahl, J., and Bentley, R. A., 2006, Isotopic evidence for mobility and group organization among Neolithic farmers at Talheim, Germany, 5000 bc, European Journal of Archaeology, 9, 259-84.

Richards, M. P., and Hedges, R. E. M., 1999, Stable isotope evidence for similarities in the types of marine foods used by Late Mesolithic humans at sites along the Atlantic coast of Europe, Journal of Archaeological Science, 26, 717-22.

Reynard, L. M., and Hedges, R. E. M., 2008, Stable hydrogen isotopes of bone collagen in palaeodietary and palaeoenvironmental reconstruction, Journal of Archaeological Science, 35(7), 1934-42.

Rovira Buendía, N., 2007, Agricultura y gestión de los recursos vegetales en el sureste de la península ibérica durante la prehistoria reciente, $\mathrm{PhD}$ thesis, Universitat Pompeu Fabra, Barcelona.

Robb, J., Bigazzi, R., Lazzarini, L., Scarsini, C., and Sonego, F., 2001, Social 'status' and biological 'status': a comparison of grave goods and skeletal indicators, American Journal of Physical Anthropology, 115, $213-22$.

Sangmeister, E., and Schubart, H., 1981, Zambujal, die Grabungen 1964 bis 1973, Philipp von Zabern, Mainz am Rhein. 
Schoeninger, M. J., and Moore, K., 1992, Bone stable isotope studies in archaeology, Journal of World Prehistory, 6(2), 247-96.

Schulting, R. J., and Richards, M. P., 2001, Dating women and becoming farmers: new palaeodietary and AMS dating evidence from the Breton Mesolithic cemeteries of Teviec and Hoedic, Journal of Anthropological Archaeology, 20(3), 314-44.

Schwarcz, H. P., 2000, Some biochemical aspects of carbon isotopic paleodiet studies, in Biogeochemical approaches to paleodietary analysis (eds. S. H. Ambrose and M. A. Katzenberg), 189-209, Kluwer Academic/Plenum Publishers, New York.

Silva, A. M., 2002, Antropologia funeraria e paleobiologia das populações portuguesas (litorais) do Neolitico final/ Calcolitico, $\mathrm{PhD}$ thesis, Universidade de Coimbra, Coimbra.

Silva, A. M., 2003, Portuguese populations of Late Neolithic and Chalcolithic periods exhumed from collective burials: an overview, Anthropologie, 1-2, 55-64.

Smith, B. N., and Epstein, S., 1971, Two categories of ${ }^{13} \mathrm{C} /{ }^{12} \mathrm{C}$ ratios for higher plants, Plant Physiology, 47, 380-4.

Spindler, K., 1981, Cova da Moura: die Besiedlung des atlantischen Küstengebietes Mittelportugals vom Neolithikum bis an das Ende der Bronzezeit, Philipp von Zabern, Mainz am Rhein.

Strickland, S., and Shetty, P., 1998, Human biology and social inequality, in Human biology and social inequality (eds. R. Strickland and P.S. Shetty), Cambridge University Press, Cambridge.

Stuart-Williams, H. L. Q., Schwarcz, H. P., White, C. D., and Spence, M. W., 1996, The isotopic composition and diagenesis of human bone from Teotihuacan and Oaxaca, Mexico, Palaeogeography, Palaeoclimatology, Palaeoecology, 126(1-2), 1-14.

Tafuri, M. A., Craig, O. E., and Canci, A., 2009, Stable isotope evidence for the consumption of millet and other plants in Bronze Age Italy, American Journal of Physical Anthropology, 139, 146-53.

Tauber, H., 1983, ${ }^{14} \mathrm{C}$ dating of human beings in relation to dietary habits, PACT, 8, 365-75.

Thomas, J., 2003, Thoughts on the 'repacked' Neolithic revolution, Antiquity, 77(295), 67-74.

Thomas, J. T., 2011, Fashioning identities, forging inequalities: Late Neolithic/Copper Age personal ornaments of the Portuguese Estremadura, European Journal of Archaeology, 14(1-2), 29-59.

Trindade, L., and da Veiga Ferreira, O., 1956, A necrópole do Cabeço da Arruda (Torres Vedras), Anais da Faculdade de Ciências do Porto, 38(4), 195-212.

Tykot, R. H., 2002, Contribution of stable isotope analysis to understanding dietary variation among the Maya, in Archaeological chemistry: materials, methods, and meanings (ed. K. A. Jakes), 214-30, American Chemical Society, Washington, DC.

Tykot, R. H., 2004, Stable isotopes and diet: you are what you eat, in Physics methods in archaeometry: proceedings of the International School of Physics 'Enrico Fermi' course CLIV (eds. M. M. Milazzo and M. Piacentini), 433-44, Società Italiana di Fisica, Bologna.

Tykot, R. H., 2006, Isotope analyses and the histories of maize, in Histories of maize: multidisciplinary approaches to the prehistory, linguistics, biogeography, domestication, and evolution of maize (eds. J. E. Staller, R. H. Tykot and B. F. Benz), 131-42, Elsevier Academic Press, Amsterdam.

Uerpmann, H., and Uerpmann, M., 2003, Zambujal: Die Stein- und Beinartefakte aus den Grabungen 1964 bis 1973 , Madrider Beiträge Band 5, Zambujal Teil 4, Philipp von Zabern, Mainz am Rhein.

Van Strydonck, M., Boudini, M., and Ervynck, A., 2002, Stable isotopes and diet: animal and human bone collagen from prehistoric sites on Mallorca, Menorca, and Formentera (Balearic Islands, Spain), in World islands in prehistory (eds. W. H. Waldren and J. A. Ensenyat), 189-97, Archaeopress, Oxford.

Warinner, C., and Tuross, N., 2010, Brief communication. Tissue isotopic enrichment associated with growth depression in a pig: implications for archaeology and ecology, American Journal of Physical Anthropology, 141(3), 486-93.

Waterman, A. J., 2006, Health status in prehistoric Portugal: dental pathology and childhood mortality patterns from the Late Neolithic burials of Feteira (Lourinhã), MA thesis, University of Iowa, Iowa City.

Waterman, A. J., 2012, Marked in life and death: identifying biological markers of social differentiation in late prehistoric Portugal, $\mathrm{PhD}$ thesis, University of Iowa, Iowa City.

Waterman, A. J., and Thomas, J. T., 2011, When the bough breaks: childhood mortality and burial practice in late Neolithic Atlantic Europe, Oxford Journal of Archaeology, 30(2), 165-83.

Waterman, A. J., Silva, A. M., and Tykot, R. H., 2014a, Stable isotopic indicators of diet from two late prehistoric burial sites in Portugal: an investigation of dietary evidence of social differentiation, Open Journal of Archaeometry, 2, 227.

Waterman, A. J., Peate, D. W., Silva, A. M., and Thomas, J. T., 2014b, In search of homelands: using strontiumisotopes to identify biological markers of mobility in late prehistoric Portugal, Journal of Archaeological Science, 42C, 11927. 
Waters-Rist, A. L., and Katzenberg, M. A., 2010, The effect of growth on stable nitrogen isotope ratios in subadult bone collagen, International Journal of Osteoarchaeology, 20(2), 172-91.

White, C. D., Spence, M. W., and Longstaffe, F. J., 2004, Demography and ethnic continuity in the Tlailotlacan enclave of Teotihuacan: the evidence from stable oxygen isotopes, Journal of Anthropological Archaeology, 23, 385-403.

Wiessner, P., and Schiefenhovel, W. (eds.), 1996, Food and the status quest: an interdisciplinary perspective, Berghahn Books, Providence, RI.

Wright, L. E., and Schwarcz, H.P., 1998, Stable carbon and oxygen isotopes in human tooth enamel: identifying breastfeeding and weaning in prehistory, American Journal of Physical Anthropology, 106(1), 1-18. 